\title{
Some Important Features of Manganites
}

\author{
R. J. Singh \\ Physics Department, Aligarh Muslim University, Aligarh, India \\ Email: ranajsingh@yahoo.com
}

Received April 12, 2012; revised September 7, 2012; accepted October 13, 2012

\begin{abstract}
Manganites are very complex systems because of interplay among charge, spin, orbital and lattice degrees of freedom. To come closer to the understanding of its nature, we discuss its three important features: 1) correlation between magnetization and electrical resistivity in the same temperature range; 2) detection of chemical constitution and the arrangement of $\mathrm{Mn}^{3+}$ and $\mathrm{Mn}^{4+}$ ions at different hole concentrations; and 3) how electrical current flows through double exchange in manganites. The first feature will be discussed for three-dimensional manganies. The features 2 and 3 are inscrutable in three-dimensional manganites. So they will be discussed for one-dimensional manganites and then generalized. One-dimensional solid has been discussed because it may give a see-through picture of various aspects of manganites. All the discussions will be done through a representative example of $\mathrm{La}_{1-\mathrm{x}} \mathrm{Ca}_{\mathrm{x}} \mathrm{MnO}_{3}$, because it is the intermediate bandwidth manganite; has been most widely investigated and has the highest magnetoresistance. If two things: 1) magnetization and resistivity as a function of temperature at various magnetic fields; and 2) phase diagrams of other manganites are known, their properties can be understood by the discussion of the three features mentioned above.
\end{abstract}

Keywords: Manganites; Electrical Resistivity; Magnetization; Phase Diagram

\section{Introduction}

Manganites $\left(\operatorname{Re}_{1-\mathrm{x}} \mathrm{M}_{\mathrm{x}} \mathrm{MnO}_{3}\right)$, where $\mathrm{Re}=\mathrm{La}, \mathrm{Y}, \mathrm{Pr} . \mathrm{Nd}$, etc. and $\mathrm{M}=\mathrm{Ca}, \mathrm{Sr}, \mathrm{Ba}, \mathrm{Pb}$, etc. have claimed wide attention due to the property of colossal magnetoresistance (CMR) and tantalizing prospect of practical applications and are also important from the point of view of microscopic understanding. Manganites are very complex systems. Probably all degrees of freedom conceivable in condensed matter physics are present here e.g., lattice, orbital, spin, charge and interplay among them, crystal structure differences, crystal defects, single crystal, polycrystalline and amorphous materials, different kinds of magnetic states-ferromagnetic (FM), antiferromagnetic (AFM), canted antiferromagnetic (CAFM), paramagnetic (PM), mixture of magnetic states or spin glass, phase coexistence, phase separation, phase coherence, inhomogeneities (large and small, temporal and spatial), pseudogaps, grain size, intergrain disorder, grain surface anomalies, tolerance factor, single and multilayer compounds, small, intermediate and large bandwidths, double exchange(DE) and superexchange (SE) interactions, Coulomb and Jahn-Teller interactions, different hole concentrations, different spin stiffness constants, different substituents for $\mathrm{Re}$ and $\mathrm{M}$ in the formula unit, conductor (half metallic), insulator (half insulator), known and unknown impurities, effect of temperature, pressure, magnetic and electric fields and their directionalities and above all CMR and what not. Large number [1-15] of theoretical and experimental works have been carried out but satisfactory answer to the properties and characteristics of manganites have not been obtained as yet.

With the help of some important experimental results given in $[16,17]$, we have tried to understand several important features of manganites in simpler ways. These features are the following: 1) to find correlation between electrical resistivity and magnetization in the same temperature range at different hole concentrations (x-values); 2) to explore chemical constitution and distribution of $\mathrm{Mn}^{3+}$ and $\mathrm{Mn}^{4+}$ ions (which mainly decide the electrical and magnetic properties of manganites) for different $\mathrm{x}$-values; 3) to understand the way the electric current flows through manganites by means of DE mechanism [18]. The first important feature e.g., correlation between electrical resistivity and magnetization will be obtained with the help of the data from three-dimensional (3-D) manganites. But the last two features seem to be more mysterious. Hence these two problems (a) chemical constitution and distribution of $\mathrm{Mn}^{3+}$ and $\mathrm{Mn}^{4+}$ in the sample will be explored through one dimensional (1-D) manganite and then generalized. In the problem (a), chemical constitution and arrangement of ions $\mathrm{Mn}^{3+}$ and $\mathrm{Mn}^{4+}$ ions in 1-D manganites will be proposed for different $\mathrm{x}$-values in such a manner that that their magnetic and electrical characteristics (FM metal, AFM insulator, FM insulator 
and also electrical resistivity etc.) come out in the same proportion as in the phase diagram (PD) of 3-D manganite [in the present case it is compared with $\mathrm{La}_{1-\mathrm{x}} \mathrm{Ca}_{\mathrm{x}} \mathrm{MnO}_{3}$ (LCMO)]. The important thing in the problem (b) is to show how the directions of the spins of ions (if parallel to the applied magnetic field, denoted by the symbol " $u$ " and if antiparallel, denoted by the symbol "d") connected by SE interaction in an 1-D chain of manganite are affected when DE jump of electrons takes place from $\mathrm{Mn}^{3+}$ to $\mathrm{Mn}^{4+}$ any where in the linear chain.

\section{Resistivity versus Magnetization}

Schiffer et al. [16] studied magnetization (M) and electrical resistivity $(\rho)$ and magnetoresistance $(\mathrm{MR})$ of $\mathrm{La}_{0.75}$ $\mathrm{Ca}_{0.25} \mathrm{MnO}_{3}$ as a function of temperature and magnetic field and the results were plotted in a figure. Part of this figure has been reproduced here as Figure 1. Khan and Singh [15] on examining this figure had indicated inverse proportionality between temperature dependence of magnetization and electrical resistivity up to the Curie temperature (Tc). This problem has been visited again here to demonstrate inverse proportionality between temperature dependence of $\mathrm{M}$ and $\rho$ (rho) in a more convincing way by superposing graphs of $1 / \mathrm{M}$ and $\rho$ under the same magnetic field and in the same temperature range from near zero Kelvin to Tc. Above Tc upto $350 \mathrm{~K}$ (highest temperature in this experiment), direct proportionality between $\mathrm{M}$ and $\rho$ has been observed and is

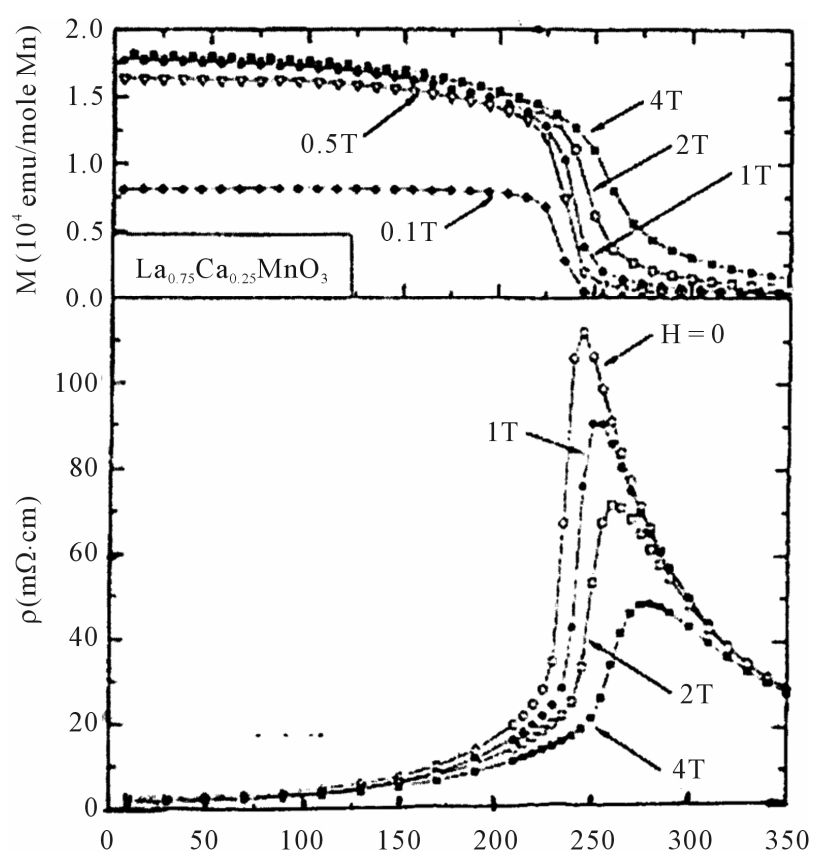

Figure 1. Magnetization and resistivitty of $\mathrm{La}_{0.75} \mathrm{Ca}_{0.25} \mathrm{MnO}_{3}$ as a function of temperature at various magnetic fields [It is a part of the figure from $P$. Schiffer et al., Physical Review Letters 75, (1995) 3336-3339]. demonstrated by superposing graphs of $\mathrm{M}$ and $\rho$, both carried out under the same strength of magnetic field. Before plotting $\mathrm{M}$ and $\rho$ curves together to show direct and inverse proportionality, height of both the $\mathrm{M}$ and $\rho$ curves were scaled to the same magnitude as the length of both the curves was already the same. Curves between $1 / \mathrm{M}$ and $\rho$ have been shown in the Figures 2(a)-(c) and between $\mathrm{M}$ and $\rho$ in the Figures 2(d)-(f) at different magnetic fields. On close inspection of the Figures 2(a)(f), it becomes clear that at higher magnetic fields, coincidence between $1 / \mathrm{M}$ and $\rho$ before Tc and between $\mathrm{M}$ and $\rho$ beyond Tc becomes better.

First we explain inverse proportionality between $M$ and $\rho$ shown in the Figures 2(a)-(c). It is generally agreed that electrical conductivity $(1 / \rho)$ is governed by Zener double exchange [18] in which the velocity of the electrons is decided by the relative direction of spins of electrons in the consecutive $\mathrm{Mn}^{3+}\left(\mathrm{t}_{2 \mathrm{~g}}^{3} \mathrm{e}_{\mathrm{g}}^{1}\right)$ and $\mathrm{Mn}^{4+}\left(\mathrm{t}_{2 \mathrm{~g}}^{3}\right)$ ions, being maximum when spins of both the ions are parallel to each other. Thus at very low temperatures, the conductivity is maximum or the resistivity is minimum. As the temperature increases, alignment of spins is disturbed; perhaps the spins (considering classical nature of spins) start oscillating about a mean position with different phase angles. Consequently, the transport of electrons is slowed down because of the mismatching of spin directions, as a result of which resistivity starts increasing. When the temperature approaches Tc, there will be more disorder in the distribution of the directions of electronic spins; thus resistivity will go on increasing with temperature and will attain a maximum at Tc. The opposite is the case for magnetization. As temperature increases, disorderliness in the parallel orientation of magnetic moments will increase resulting into decrease of net magnetization and it will become minimum at Tc. Thus temperature dependence of $\mathrm{M}$ and $\rho$ are of opposite nature.

Beyond Tc, the charge carriers in manganites are spin or magnetic polarons as concluded in [15]. In magnetic polarons, the charge carriers are surrounded by electron spins arranged in certain order. When temperature increases beyond Tc, orderliness of spins surrounding the charge carriers goes on decreasing. As the orderliness of spin environment is destroyed, their control on the movement of charge carriers is loosened and thus they can move faster resulting into the decease of resistivity. When the orderliness of spins surrounding the charge carriers is loosened by the increase of temperature, the sum total of magnetic moments in the direction of the magnetic field will also decrease. Hence beyond Tc, both $\mathrm{M}$ and $\rho$ will decrease with the rise of temperature.

On examining the Figures 2(a)-(f), it becomes clear that at higher magnetic fields, there is better correlation between $1 / \mathrm{M}$ and $\rho$ below Tc and between $\mathrm{M}$ and $\rho$ be- 
yond Tc. It has been noted in the last two paragraphs that any relationship between $\mathrm{M}$ and $\rho$ below or beyond Tc is dependent on the strength of magnetic field and hence better coincidence between $1 / \mathrm{M}$ and $\rho$ below Tc and between $\mathrm{M}$ and $\rho$ with increasing magnetic field is explained.

Not only in $\left(\mathrm{Re}_{1-\mathrm{x}} \mathrm{M}_{\mathrm{x}} \mathrm{MnO}_{3}\right)$ types of manganites, very strong correlation between $\mathrm{M}$ and $\rho$ has been found in bilayered manganites as $\left[\left(\mathrm{La}_{0.5} \mathrm{Nd}_{0.5}\right)_{1.2} \mathrm{Sr}_{1.8} \mathrm{Mn}_{2} \mathrm{O}_{7}\right]$. Daquon Liao et al. [18] noted that there are step like magnetizations and also step like resistivity jumps in the bi-layered manganite $\left[\left(\mathrm{La}_{0.5} \mathrm{Nd}_{0.5}\right)_{1.2} \mathrm{Sr}_{1.8} \mathrm{Mn}_{2} \mathrm{O}_{7}\right]$ as shown in Figure 3. In Figure 3, there are two parts, one on the left and the other on the right side. In each part,

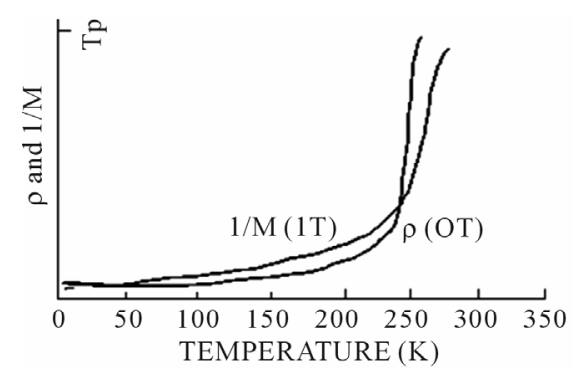

(a)

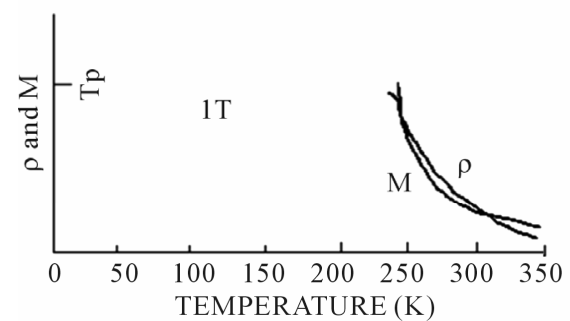

(d)

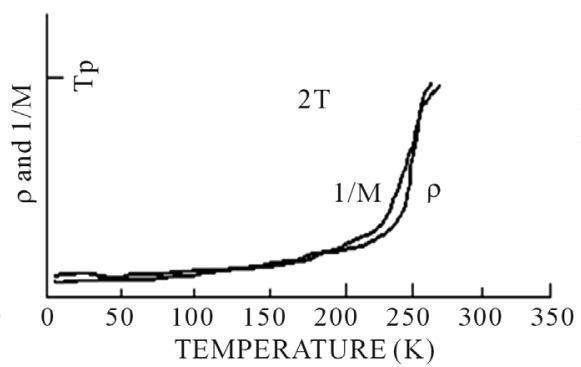

(b)

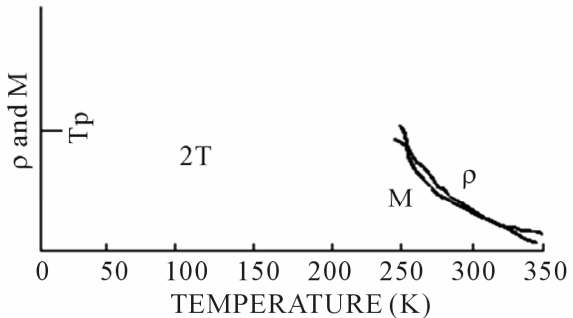

(e)

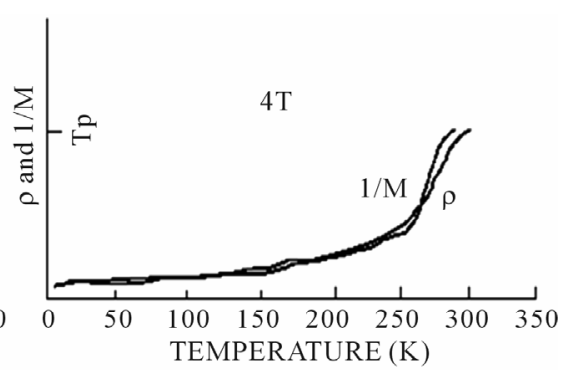

(c)

Figure 2. (a) Showing $1 / M(M=$ magnetization) versus temperature $(T)$ curve and resistivity $(\rho$ or rho) versus (T) curve together. $\rho$-T curve is at zero magnetic field, but $1 / M-T$ curve is at 1 Tesla. Magnetic field is essential for the measurement of $M$ and hence one of the lowest magnetic fields (in the measurement of $M$ ) has been chosen for comparing the two curves; (b) 1/M-T and $\rho$-T curves together, both at magnetic field of 2 Tesla; (c) 1/M-T and $\rho$-T curves together at magnetic field of 4 T (Tesla); (d)-(f) showing (M-T) and $(\rho-T)$ curves beyond $T_{p}$ (peak temperature $=250^{\circ}$ ) at 1,2 and 4 teslas respectively. All these figures have been derived from Figure 1.
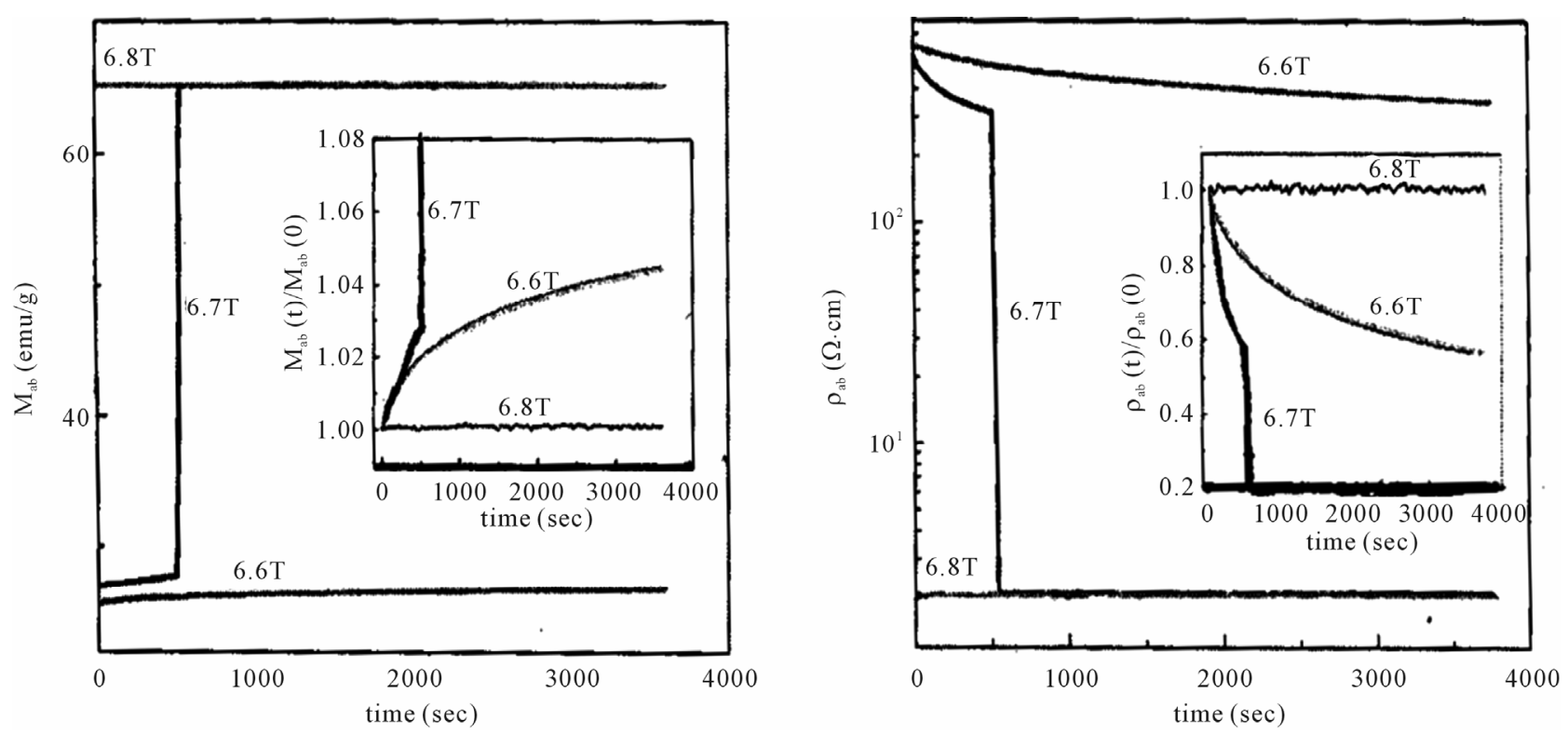

Figure 3. Step-like magnetization and resistivity jumps at magnetic fields of 6.6, 6.7 and 6.8 Tesla in bilayered $\left[\left(\mathrm{La}_{0.5} \mathrm{Nd}_{0.5}\right)_{1.2}\right.$ $\mathrm{Sr}_{1.8} \mathrm{Mn}_{2} \mathrm{O}_{7}$ ] single crystal in its ab plane at $2 \mathrm{~K}$ [Daquan Liao, Young Sun, Renfu Yang, Qing'an Li and Zhaohua Cheng, Physical Review B 74, (2006) 174434-174439]. 
there is a main section and an inset. In the main section of the left part, magnetization of $\left[\left(\mathrm{La}_{0.5} \mathrm{Nd}_{0.5}\right)_{1.2} \mathrm{Sr}_{1.8} \mathrm{Mn}_{2} \mathrm{O}_{7}\right]$ at 6.6, 6.7, 6.8 Tesla $(\mathrm{T})$ at $2 \mathrm{~K}$ has been shown. In the main section of the right part, resistivity of the same sample at the same values of magnetic fields and temperatures have been shown. Even a cursory view of these figures will unmistakably reveal inverse proportionality between magnetization and resistivity. In the inset of the left part of the figure, increase of magnetization with time after the imposition of $6.6 \mathrm{~T}$ of magnetic field has been shown. In the inset of the right part of the figure, the decrease of resistivity with time after the imposition of 6.6 T of magnetic field has been shown. In Figure 4 is shown a plot of $\mathrm{M}_{\mathrm{ab}}(\mathrm{t}) / \mathrm{M}_{\mathrm{ab}}(0)$ versus $1 /\left[\rho_{\mathrm{ab}}(\mathrm{t}) / \rho_{\mathrm{ab}}(0)\right]$. Very strong inverse proportionality between time dependence of growth of $\mathrm{M}$ and $\rho$ is clearly seen.

\section{Chemical Constitution and Arrangement of $\mathrm{Mn}^{3+}$ and $\mathrm{Mn}^{4+}$ Ions in One-Dimensional Manganites with Different Hole Concentrations}

Chemical constitution of 1-D manganite and arrangement of $\mathrm{Mn}^{3+}$ and $\mathrm{Mn}^{4+}$ ions in them for different hole concentrations(x) have been given in Table 1.

Chemical constitution of 1-D chains and arrangement of $\mathrm{Mn}^{3+}$ and $\mathrm{Mn}^{4+}$ ions in them has been chosen in such a manner that the magnetic and electrical characteristics of the 1-D chains vary in the same proportion as in the 3-D manganite shown in the phase diagram [16] of LCMO (Figure 5). It is hoped that the information obtained from 1-D manganite may help in understanding the constitution, structure and other properties of 3-D manganites. It has been found that in manganite samples, there are ordered grains separated by spans of disordered materials. In 1-D manganites also, there is expected to be chains of certain lengths with ordered arrangement of atoms/ions separated by smaller lengths of disordered material.

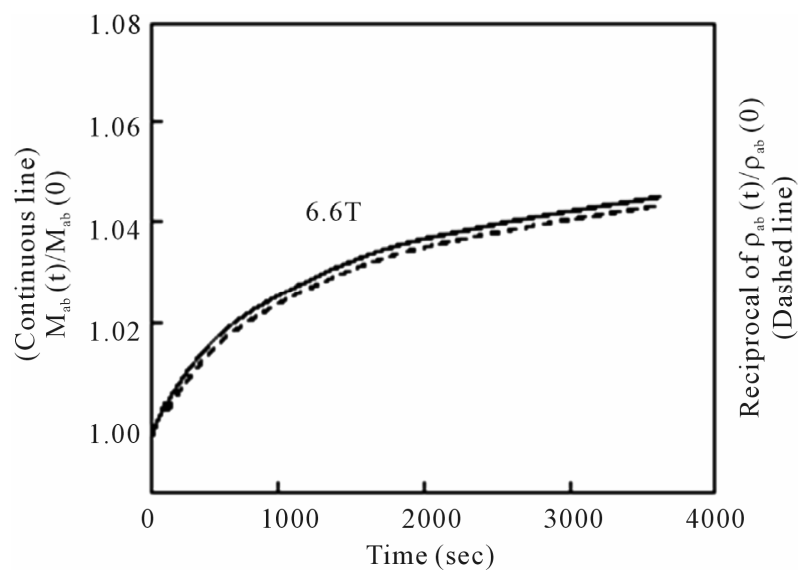

Figure 4. Showing coincidence of $\mathrm{M}_{\mathrm{ab}}(\mathrm{t}) / \mathrm{M}_{\mathrm{ab}}(0)$ and reciprocal of $\rho_{a b}(t) / \rho_{a b}(0)$. This figure is based on Figure 3.

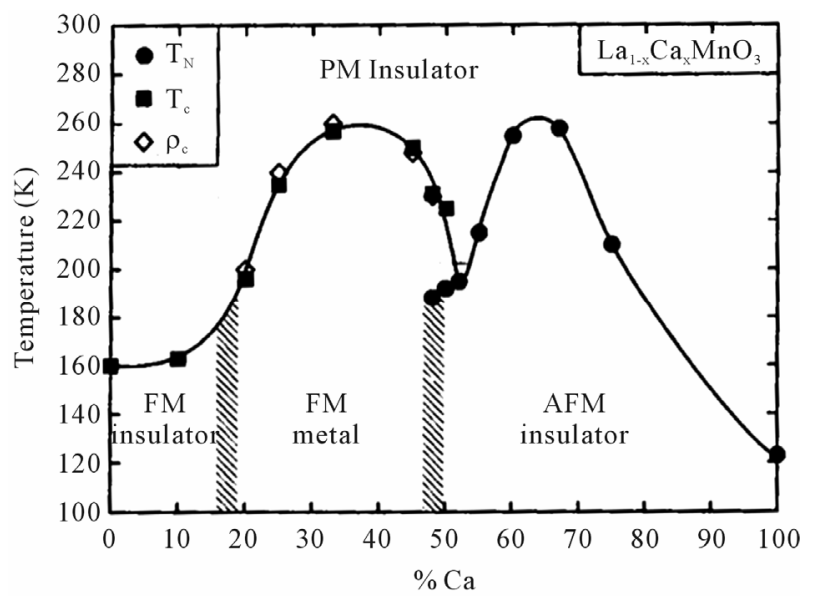

Figure 5. Phase diagram of $\mathrm{La}_{1-x} \mathrm{Ca}_{\mathrm{x}} \mathrm{MnO}_{3}$ [P. Schiffer, A. P. Ramirez, W. Bao and S.-W. Cheong, Physical Review Letters, 75 (1995) 3336-3339].

Hence in proposing structure of any specimen for any $\mathrm{x}$, the constitution and arrangement of atoms/ions only in the ordered chains will be given. The role of intervening disordered chains will be discussed later. In 1-D manganite, the ordered chain will be a linear chain of $\mathrm{Mn}^{3+}$ and $\mathrm{Mn}^{4+}$ ions. It is because of the fact that magnetic and electrical properties of manganites are mainly decided by the relative abundance of $\mathrm{Mn}^{3+}$ and $\mathrm{Mn}^{4+}$ ions and the sequence in the arrangement of these two ions. Other atoms/ions play subservient roles. As regards the number of $\mathrm{Mn}^{3+}$ and $\mathrm{Mn}^{4+}$ ions corresponding to a particular Mn-valency, their numbers are adjusted in such a manner that the particular Mn-valency is obtained. For the purpose of calculation, $\mathrm{Mn}^{3+}\left(\mathrm{t}_{2 \mathrm{~g}}^{3} \mathrm{e}_{\mathrm{g}}^{1}\right)$ has valency $=3$ and $\mathrm{Mn}^{4+}\left(\mathrm{t}_{2 \mathrm{~g}}^{3}\right)$ has valency $=4$. In the manganite $\left(\operatorname{Re}_{1-\mathrm{x}} \mathrm{M}_{\mathrm{x}}\right.$ $\left.\mathrm{Mn}_{3}\right)$, the number of $\mathrm{Mn}^{3+}$ is proportional to $(1-\mathrm{x})$ and of $\mathrm{Mn}^{4+}$ proportional to (x). In the presentation of linear chains in Table 1 and elsewhere also, for simplicity, $\mathrm{Mn}^{3+}$ will be represented as " 3 " and $\mathrm{Mn}^{4+}$ as " 4 ". Linear chains of 3 and 4 ions representing different Mn-valencies (3.0, 3.1, 3.2, 3.3, 3.4, 3.5, 3.6, 3.7, 3.8, 3.9, 4.0) have been shown in Table 1.

The principle in deciding the sequence of the ions 3 and 4 for Mn-valencies from 3.1 to 3.4 is the condition of minimum Madelung potential. For valencies 3.6 to 3.9, charge ordering is the guiding principle, according to which in the chain, first all 3 ions are placed and then all 4 ions. For Mn-valencies $=3.0$ and 4.0, there is no choice. For valency $=3.0$, all ions are 3 and for valency $=4.0$, all ions are 4 . Mn-valency $=3.5$ occupies a special position in all manganites. It has been found to have CE-configuration which means coexistence of ferromagnetism and charge ordering. In Table 1, the same rule has been applied for the Mn-valency $=3.5$.

In Table 1, ions suffixed by "u" are in the direction of the applied magnetic field and those suffixed by "d" 
Table 1. Linear chains of $\mathrm{Mn}^{3+}$ (3) and $\mathrm{Mn}^{4+}$ (4) representing different Mn-valencies. Number of spins in the direction of magnetic field (= number of spins in the upward direction $=u$ ); number of spins opposite to the direction of the magnetic field ( $=$ number of spins in the downward direction $=d$ ); number of conduction channels by double exchange $=$ ch; difference in the number of spins in the $u$ and d-directions $=\mathbf{E x}$.

\begin{tabular}{ccc}
\hline Mn-valency & \multicolumn{1}{c}{ Arrangement of ions } & u, d, ch, ex \\
\hline 3.0 & $3 u 3 d 3 u 3 d 3 u 3 d 3 u 3 d 3 u 3 d 3 u 3 d 3 u 3 d 3 u 3 d 3 u 3 d 3 u 3 d$ & $10,10,0,0$ \\
3.1 & $3 u 3 d 3 u 3 d 3 u 3 d 3 u 4 u 3 u 3 d 3 u 3 d 3 u 3 d 3 u 4 u 3 u 3 d 3 u 3 d$ & $12,8,2,4$ \\
3.2 & $3 u 3 d 3 u 4 u 3 u 3 d 3 u 4 u 3 u 3 d 3 u 3 d 3 u 4 u 3 u 3 d 3 u 4 u 3 u 3 d$ & $14,6,4,8$ \\
3.3 & $3 u 3 d 3 u 4 u 3 u 4 u 3 u 3 d 3 u 4 u 3 u 4 u 3 u 3 d 3 u 4 u 3 u 4 u 3 u 3 d$ & $16,4,6,12$ \\
3.4 & $3 u 3 d 3 u 4 u 3 u 4 u 3 u 4 u 3 u 4 u 3 u 4 u 3 u 4 u 3 u 4 u 3 u 4 u 3 u 3 d$ & $18,2,8,16$ \\
3.5 & $3 u 3 d 3 u 3 d 3 u 3 d 3 u 4 u 3 u 4 u 3 u 4 u 3 u 4 u 4 d 4 u 4 d 4 u 4 d 4 u$ & $14,6,4,8$ \\
3.6 & $3 d 3 u 3 d 3 u 3 d 3 u 3 d 3 u 4 u 4 d 4 u 4 d 4 u 4 d 4 u 4 d 4 u 4 d 4 u 4 d$ & $10,10,1,0$ \\
3.7 & $3 d 3 u 3 d 3 u 3 d 3 u 4 u 4 d 4 u 4 d 4 u 4 d 4 u 4 d 4 u 4 d 4 u 4 d 4 u 4 d$ & $10,10,1,0$ \\
3.8 & $3 d 3 u 3 d 3 u 4 u 4 d 4 u 4 d 4 u 4 d 4 u 4 d 4 u 4 d 4 u 4 d 4 u 4 d 4 u 4 d$ & $10,10,1,0$ \\
3.9 & $3 d 3 u 4 u 4 d 4 u 4 d 4 u 4 d 4 u 4 d 4 u 4 d 4 u 4 d 4 u 4 d 4 u 4 d 4 u 4 d$ & $10,10,1,0$ \\
4.0 & $4 d 4 u 4 d 4 u 4 d 4 u 4 d 4 u 4 d 4 u 4 d 4 u 4 d 4 u 4 d 4 u 4 d 4 u 4 d 4 u$ & $10,10,1,0$ \\
\hline
\end{tabular}

are in the direction opposite to the applied field. The difference in their numbers (number of u spins - number of $\mathrm{d}$ spins) in any chain is proportional to its ferromagnetic character. When number of $u$ spins $=$ number of $d$ spins, then the chain is antiferromagnetic. Number of conduction channels is proportional to the electrical conductivity of the chain or inversely proportional to the resistivity of the chain. Electrical conduction channels exist only between ions 3 and 4 when both are either in u-state or d-state. Electrons jump only between $e_{\mathrm{g}}$ levels of ions 3 and 4. Electron can jump from ion 3 to 4 by means of double exchange, but not in the reverse direction because there is no electron in the $e_{\mathrm{g}}$ level of the ion 4 .

Tsai et al. [19] have pointed out that in the FM phase shown in the PDs, the resistivity is much higher than in the metals. The resistivities of metals are generally of the order of $10^{-6} \mathrm{ohm}-\mathrm{cm}$, but in the FM region it is of the order of $1 \mathrm{ohm}-\mathrm{cm}$. Had the FM phase in the PDs been purely FM, the resistivity would have been very low. The region FM is called metallic only because its resistivity rises with temperature. The region which is denoted as AFM is not purely antiferromagnetic. Had it been purely AFM, there would not have been any electrical conduction (due to DE) and the resistivity would have been very high. In fact the resistivity in this region is of the order of $10^{2} \mathrm{ohm}-\mathrm{cm}$ near zero Kelvin, whereas in ordinary insulators, the resistivity is of the order of $10^{10} \mathrm{ohm}-\mathrm{cm}$ at room temperature.

In the linear chains given in Table 1 against every hole concentration, exact resistivity or exact magnetization or FM strength can not be estimated, but care has been taken in framing the chains that in FM region, con- ductivity (proportional to the number of channels) as well as FM strength (proportional to excess of $u$ over $\mathrm{d}$-spins) goes on increasing in going from $\mathrm{x}=0.2$ to 0.4 as observed in the PD. In AFM region, number of $\mathrm{u}$ spins $=$ number of $\mathrm{d}$ spins as required, but one conductivity channel has been kept open to account for the limited conductivity in this region.

In Table 1, chain lengths contain only 20 ions of $\mathrm{Mn}^{3+}$ and $\mathrm{Mn}^{4+}$. Experiments indicate that grain size in 3-D manganites vary from 10 to 100 nanometer. Smaller chain lengths have been taken here to get better insight into the flow of current and arrangement of ions in the chains. Chain lengths can be extended to any desired value without much difficulty. As an example, the chain length corresponding to Mn-valency $=3.2$ (see Table 1) can be made twice longer in the following two ways: (a) by writing the expression twice as " $3 \mathrm{u} 3 \mathrm{~d} 3 \mathrm{u} u 4 \mathrm{u} 3 \mathrm{u} 3 \mathrm{~d} 3 \mathrm{u}$ $4 u 3 u 3 d 3 u 3 d 3 u 4 u 3 u 3 d 3 u 4 u 3 u 3 d+3 u 3 d 3 u 4 u 3 u 3 d 3 u 4 u 3 u$ $3 \mathrm{~d} 3 \mathrm{u} 3 \mathrm{~d} 3 \mathrm{u} 4 \mathrm{u} 3 \mathrm{u} 3 \mathrm{~d} 3 \mathrm{u} 4 \mathrm{u} 3 \mathrm{u} 3 \mathrm{~d}$ " and (b) as " $3 \mathrm{u} 3 \mathrm{~d} 3 \mathrm{u} 3 \mathrm{~d} 3 \mathrm{u} 4 \mathrm{u}$ $3 u 4 u 3 u 3 d 3 u 3 d 3 u 4 u 3 u 4 u 3 u 3 d 3 u 3 d 3 u 3 d 3 u 3 d 3 u 4 u 3 u 4 u 3 u$ $3 d 3 u 3 d 3 u 4 u 3 u 4 u 3 u 3 d 3 u 3 d "$ by doubling each segment but always keeping doubled pair of $3 u 4 u$ together in the chain.

In the chain corresponding to Mn-valency $=3.2$ in Table 1, we have 14 spins in u-state and 6 in the d-state, 4 conductivity channels and ex $=8$. When the chain length is doubled in either of the two ways, (a) or (b); these characteristic numbers are also doubled i.e., 28 u-states, $12 \mathrm{~d}$-states; 8 conductivity channels and ex $=16$. The above example shows that the chain length can be increased any number of times to match the size of the real crystals. 
Another important thing to be noted is that the interaction between adjacent ions is of super-exchange type as was established by Anderson [20] in the early days of manganites. Thus the interaction between the nearest Mn-ions in Table 1, has been decided by the rules of SE interaction. Only nearest neighbour exchange interaction has been considered. SE interaction is expressed in terms of Goodenough-Kanamori [21-23] semi-empirical rules. We will point out only those rules which have been used here. They are: 1) The SE interaction is antiferromagnetic (spin directions reversed in the interacting ions i.e., from $u$ to $d$ or from $d$ to $u$ ) between two magnetic ions with half filled orbital which couple through a nonmagnetic ion (as $\mathrm{O}^{2-}$ ), which means that interaction between $\mathrm{Mn}^{3+}$ and $\mathrm{Mn}^{3+}$ or between 3 and 3 will be of $\mathrm{AF}$ type. 2) Coupling between a half-filled orbital and a vacant orbital is ferromagnetic i.e., interaction between $\mathrm{Mn}^{3+}$ and $\mathrm{Mn}^{4+}$ or between $\mathrm{Mn}^{4+}$ and $\mathrm{Mn}^{3+}$ (or between 3 and 4 or between 4 and 3) will be ferromagnetic. The ferromagnetic interaction means that the spin directions of both the interacting ions will be the same (both being "u" or both being "d"). 3) SE interaction between two empty orbitals provided each cation carries a net spin and empty orbitals share the anion p-orbitals will be antiferromagnetic, which means that the interaction between $\mathrm{Mn}^{4+}$ and $\mathrm{Mn}^{4+}$ or between 4 and 4 will be antiferromagnetic.

\section{Double Exchange Mechanism in Linear Chains}

We will show how electric current flows through a 1-D manganite by DE mechanism. According to DE, an electron jumps from $\mathrm{Mn}^{3+}$ to $\mathrm{O}^{2-}$ and at the same time an electron jumps from $\mathrm{O}^{2-}$ to $\mathrm{Mn}^{4+}$ thus completing transfer of an electron from $\mathrm{Mn}^{3+}$ to $\mathrm{Mn}^{4+}$. In Table 2, we will show the flow of electric current in LCMO with $\mathrm{x}=0.3$, whose composition has been given in Table 1. In the first row of Table 2, composition of the manganite consisting of total of $20 \mathrm{Mn}^{3+}$ (3) and $\mathrm{Mn}^{4+}$ (4) ions with different $\mathrm{u}$ and $\mathrm{d}$ direction of spins has been given. The second row represents the arrangement and directions of spins of ions (u or d) after DE has taken place in all the possible pairs in the first row. As a matter of fact, any row represents the arrangement and directions of spins of ions after DE has taken place in all the possible pairs in the previous row. Table 2 extends upto 13 rows because in the $13^{\text {th }}$ row there are no pairs of $3 \mathrm{u}-4 \mathrm{u}$ ions left to allow any further DE jump. As seen in Table 2, in going from one row to the next row after executing DE jumps in $3 u-4 u$ pairs, a new pair of $3 u-4 u$ is generated displaced one place to the left side of the table. But this process is disturbed on reaching near the left or the lower boundary of the table as can be verified by $3 \mathrm{u}-4 \mathrm{u}$ pairs in the row 1 and columns 3, 4; row 1 and columns 9, 10 and row 1 and columns 15, 16. Their march have been shown

Table 2. Electrical conduction in the linear chain corresponding to the Mn-valency $=3.3$. All nearest neighbour spins in a row are connected by super-exchange interaction. Double exchange electron jump takes place only between ions 3 and 4 both being in the same direction " $u$ ". The spin state of ions in a row is decided with respect to any $3 u-4 u$ pair in the row by the rules of super-exchange interaction. Symbols $u$, d, ch have the same meanings as in Table 1. All double exchange electron jumps are between $3 u$ and $4 u$ ions and are denoted here as uu type. $C=$ column number and $R=$ row number.

\begin{tabular}{|c|c|c|c|c|c|c|c|c|c|c|c|c|c|c|c|c|c|c|c|c|c|}
\hline $\mathrm{R}^{\mathrm{C}}$ & 1 & 2 & 3 & 4 & 5 & 6 & 7 & 8 & 9 & 10 & 11 & 12 & 13 & 14 & 15 & 16 & 17 & 18 & 19 & 20 & $\mathrm{u}, \mathrm{d}, \mathrm{ch}$, type \\
\hline 1 & $3 u$ & $3 d$ & $3 u$ & $4 u$ & $3 u$ & $4 u$ & $3 u$ & $3 d$ & $3 u$ & $4 u$ & $3 u$ & $4 u$ & $3 u$ & $3 d$ & $3 u$ & $4 u$ & $3 u$ & $4 u$ & $3 u$ & $3 d$ & $16,4,6,--$ \\
\hline 2 & $3 d$ & $3 u$ & $4 u$ & $3 u$ & $4 u$ & $3 u$ & $3 d$ & $3 u$ & $4 u$ & $3 u$ & $4 u$ & $3 u$ & $3 d$ & $3 u$ & $4 u$ & $3 u$ & $4 u$ & $3 u$ & $3 d$ & $3 u$ & $16,4,6, \mathrm{uu}$ \\
\hline 3 & $3 u$ & $4 u$ & $3 u$ & $4 u$ & $3 u$ & $3 d$ & $3 u$ & $4 u$ & $3 u$ & $4 u$ & $3 u$ & $3 d$ & $3 u$ & $4 u$ & $3 u$ & $4 u$ & $3 u$ & $3 d$ & $3 u$ & $3 d$ & $16,4,6, \mathrm{uu}$ \\
\hline 4 & $4 u$ & $3 u$ & $4 u$ & $3 u$ & $3 d$ & $3 u$ & $4 u$ & $3 u$ & $4 u$ & $3 u$ & $3 d$ & $3 u$ & $4 u$ & $3 u$ & $4 u$ & $3 u$ & $3 d$ & $3 u$ & $3 d$ & $3 u$ & $16,4,5, \mathrm{uu}$ \\
\hline 5 & $4 d$ & $4 u$ & $3 u$ & $3 d$ & $3 u$ & $4 u$ & $3 u$ & $4 \mathrm{u}$ & $3 u$ & $3 d$ & $3 u$ & $4 u$ & $3 u$ & $4 u$ & $3 u$ & $3 d$ & $3 u$ & $3 d$ & $3 u$ & $3 d$ & $14,6,4, \mathrm{uu}$ \\
\hline 6 & $4 u$ & $4 d$ & $3 d$ & $3 u$ & $4 u$ & $3 u$ & $4 u$ & $3 u$ & $3 d$ & $3 u$ & $4 \mathrm{u}$ & $3 u$ & $4 u$ & $3 u$ & $3 d$ & $3 u$ & $3 d$ & $3 u$ & $3 d$ & $3 u$ & $14,6,4, \mathrm{uu}$ \\
\hline 7 & $4 d$ & $4 u$ & $3 u$ & $4 \mathrm{u}$ & $3 u$ & $4 u$ & $3 u$ & $3 d$ & $3 u$ & $4 \mathrm{u}$ & $3 u$ & $4 u$ & $3 u$ & $3 d$ & $3 u$ & $3 d$ & $3 u$ & $3 d$ & $3 u$ & $3 d$ & $14,5,4$, uu \\
\hline 8 & $4 u$ & $4 d$ & $4 u$ & $3 u$ & $4 u$ & $3 u$ & $3 d$ & $3 u$ & $4 u$ & $3 u$ & $4 \mathrm{u}$ & $3 u$ & $3 d$ & $3 u$ & $3 d$ & $3 u$ & $3 d$ & $3 u$ & $3 d$ & $3 u$ & $14,6,3, \mathrm{uu}$ \\
\hline 9 & $4 d$ & $4 u$ & $4 d$ & $4 u$ & $3 u$ & $3 d$ & $3 u$ & $4 u$ & $3 u$ & $4 \mathrm{u}$ & $3 u$ & $3 d$ & $3 u$ & $3 d$ & $3 u$ & $3 d$ & $3 u$ & $3 d$ & $3 u$ & $3 d$ & $12,8,2, \mathrm{uu}$ \\
\hline 10 & $4 u$ & $4 d$ & $4 u$ & $4 d$ & $3 d$ & $3 u$ & $4 u$ & $3 u$ & $4 u$ & $3 u$ & $3 d$ & $3 u$ & $3 d$ & $3 u$ & $3 d$ & $3 u$ & $3 d$ & $3 u$ & $3 d$ & $3 u$ & $12,8,2, \mathrm{uu}$ \\
\hline 11 & $4 d$ & $4 u$ & $4 d$ & $4 u$ & $3 u$ & $4 u$ & $3 u$ & $4 \mathrm{u}$ & $3 u$ & $3 d$ & $3 u$ & $3 d$ & $3 u$ & $3 d$ & $3 u$ & $3 d$ & $3 u$ & $3 d$ & $3 u$ & $3 d$ & $12,8,2, \mathrm{uu}$ \\
\hline 12 & $4 u$ & $4 d$ & $4 u$ & $4 d$ & $4 u$ & $3 u$ & $4 u$ & $3 u$ & $3 d$ & $3 u$ & $3 d$ & $3 u$ & $3 d$ & $3 u$ & $3 d$ & $3 u$ & $3 d$ & $3 u$ & $3 d$ & $3 u$ & $12,8,1, \mathrm{uu}$ \\
\hline 13 & $4 d$ & $4 u$ & $4 d$ & $4 u$ & $4 d$ & $4 u$ & $3 u$ & $3 d$ & $3 u$ & $3 d$ & $3 u$ & $3 d$ & $3 u$ & $3 d$ & $3 u$ & $3 d$ & $3 u$ & $3 d$ & $3 u$ & $3 d$ & $10,10,0,--$ \\
\hline
\end{tabular}


by slanting lines in the table (by solid lines till $3 u-4 u$ pairs are maintained and by dotted lines, when they are disturbed). But changes observed in the $3 \mathrm{u}-4 \mathrm{u}$ pair is not something inherent. It is solely due to the limitation on the length of the chain. Had it been infinite or of very big length, such changes would not have occurred or would have been inconsequential. What is important in the table is that in the last row all the $\mathrm{Mn}^{3+}$ (or 3) ions (with one electron in the $\mathrm{e}_{\mathrm{g}}$ level) have collected on the right hand side of the table and all the $\mathrm{Mn}^{4+}$ (4) ions have collected on the left hand side, which means that electron current has flown in the right hand side of the table. In this table, it needs to be specially mentioned that DE electron jump is always between $3 \mathrm{u}$ to $4 \mathrm{u}$ ion and directions of spins of other ions in each row is adjusted according to the rules of SE interaction with respect to any $3 \mathrm{u}-4 \mathrm{u}$ ion pair in that row.

There can be other ways in which the $\mathrm{u}$ - and d-directions of the spins can be adjusted. One method is that the direction of the spin of the first ion on the left side of each row is assigned $u$ direction and the directions of all the other ions in that row is adjusted with respect to this ion according to the rules of SE interaction. Following this rule, Table 3 has been prepared for the same Mnvalency $=3.3$. In this table also, electrons (ion 3) have collected on the right hand side and ions 4 have collected on the left hand side showing that electron current has flown to the right hand side. Limitations on the length of the chain has similar effects here also. But what seems to be not acceptable is that from one row to the next, spin directions of ions mostly change from $u$ to $d$. This can be checked by proceeding along the slanting lines starting from row 1 and columns 8,9 or from row 1 and columns 17,18 . On applying the unidirectional magnetic field, as is done in the experiments, this kind of change of spin directions does not seem to be reasonable. Hence Table 2 represents the correct way in which the electric current flows in a 1-D manganite.

There is another point to be considered in discussing the flow of current through a chain. In 3-D manganites, there are supposed to be FM metallic islands separated by insulating regions. In these manganites, current through metallic islands flows through intrinsic conduction and through insulating regions by spin polarized tunnelling [24] or by opening temperature and magnetic field dependent new channels [25]. In 1-D manganites also, chains can not be of infinite lengths and there should be ordered chain lengths intervened by smaller chain lengths which have no order and can be insulating or less conducting. So in 1-D manganites also there should be intrinsic flow through the ordered portion of the chain and extrinsic flow through the disordered chains governed by the same mechanisms given in [24,25].

The important question that remains to be answered is how the study of 1-D manganite will help in understanding the properties and characteristics of 3-D manganites?

Table 3. Electrical conduction in the linear chain corresponding to the Mn-valency $=3.3$. The spin states of ions in a row are decided in a different way from that of Table 2 . Here the spin states ( $u$ or d) of ions in a row is decided by the state of the ion occurring in the first column of each row (always taking it to be in u-state) by the rules of superexchange interaction. Symbols have the same meanings as in Tables 1 and 2 . Type uu denotes DE jump between $3 \mathrm{u}$ and $4 \mathrm{u}$; dd represents DE jump between $3 d$ and $4 d$. $C=$ column number, $R=$ row.

\begin{tabular}{|c|c|c|c|c|c|c|c|c|c|c|c|c|c|c|c|c|c|c|c|c|c|}
\hline${ }^{C}$ & 1 & 2 & 3 & 4 & 5 & 6 & 7 & 8 & 9 & 10 & 11 & 12 & 13 & 14 & 15 & 16 & 17 & 18 & 19 & 20 & $\mathrm{u}, \mathrm{d}, \mathrm{ch}$, type \\
\hline 1 & $3 u$ & $3 d$ & $3 u$ & $4 u$ & $3 u$ & $4 u$ & $3 u$ & $3 d$ & $3 u$ & $4 u$ & $3 u$ & $4 u$ & $3 u$ & $3 d$ & $3 u$ & $4 u$ & $3 u$ & $4 u$ & $3 u$ & $3 d$ & $16,4,6,--$ \\
\hline 2 & $3 u$ & $3 d$ & $4 d$ & $3 d$ & $4 d$ & $3 d$ & $3 u$ & $3 d$ & $4 d$ & $3 d$ & $4 d$ & $3 d$ & $3 u$ & $3 d$ & $4 d$ & $3 d$ & $4 d$ & $3 d$ & $3 u$ & $3 d$ & $8,12,6 . d d$ \\
\hline 3 & $3 u$ & $4 u$ & $3 u$ & $4 u$ & $3 u$ & $3 d$ & $3 u$ & $4 u$ & $3 u$ & $4 u$ & $3 u$ & $3 d$ & $3 u$ & $4 u$ & $3 u$ & $4 u$ & $3 u$ & $3 d$ & $3 u$ & $3 d$ & $16,4,6, \mathrm{uu}$ \\
\hline 4 & $4 u$ & $3 u$ & $4 u$ & $3 u$ & $3 d$ & $3 u$ & $4 u$ & $3 u$ & $4 u$ & $3 u$ & $3 d$ & $3 u$ & $4 u$ & $3 u$ & $4 u$ & $3 u$ & $3 d$ & $3 u$ & $3 d$ & $3 u$ & $16,4,5, \mathrm{uu}$ \\
\hline 5 & $4 u$ & $4 d$ & $3 d$ & $3 u$ & $3 d$ & $4 d$ & $3 d$ & $4 d$ & $3 d$ & $3 u$ & $3 d$ & $4 d$ & $3 d$ & $4 d$ & $3 d$ & $3 u$ & $3 d$ & $3 u$ & $3 d$ & $3 u$ & $6,14,4, \mathrm{dd}$ \\
\hline 6 & $4 u$ & $4 d$ & $3 d$ & $3 u$ & $4 u$ & $3 u$ & $4 \mathrm{u}$ & $3 u$ & $3 d$ & $3 u$ & $4 u$ & $3 u$ & $4 u$ & $3 u$ & $3 d$ & $3 u$ & $3 d$ & $3 u$ & $3 d$ & $3 u$ & $14,6,4, \mathrm{uu}$ \\
\hline 7 & $4 u$ & $4 d$ & $3 d$ & $4 d$ & $3 d$ & $4 d$ & $3 d$ & $3 u$ & $3 d$ & $4 d$ & $3 d$ & $4 d$ & $3 d$ & $3 u$ & $3 d$ & $3 u$ & $3 d$ & $3 u$ & $3 d$ & $3 u$ & $6,14,4, \mathrm{dd}$ \\
\hline 8 & $4 u$ & $4 d$ & $4 u$ & $3 u$ & $4 u$ & $3 u$ & $3 d$ & $3 u$ & $4 u$ & $3 u$ & $4 u$ & $3 u$ & $3 d$ & $3 u$ & $3 d$ & $3 u$ & $3 d$ & $3 u$ & $3 d$ & $3 u$ & $14,6,3, \mathrm{uu}$ \\
\hline 9 & $4 u$ & $4 d$ & $4 u$ & $4 d$ & $3 d$ & $3 u$ & $3 d$ & $4 d$ & $3 d$ & $4 d$ & $3 d$ & $3 u$ & $3 d$ & $3 u$ & $3 d$ & $3 u$ & $3 d$ & $3 u$ & $3 d$ & $3 u$ & $8,12,2, \mathrm{dd}$ \\
\hline 10 & $4 u$ & $4 d$ & $4 u$ & $4 d$ & $3 d$ & $3 u$ & $4 u$ & $3 u$ & $4 u$ & $3 u$ & $3 d$ & $3 u$ & $3 d$ & $3 u$ & $3 d$ & $3 u$ & $3 d$ & $3 u$ & $3 d$ & $3 u$ & $12,8,2 . \mathrm{uu}$ \\
\hline 11 & $4 u$ & $4 d$ & $4 u$ & $4 d$ & $3 d$ & $4 d$ & $3 d$ & $4 d$ & $3 d$ & $3 u$ & $3 d$ & $3 u$ & $3 d$ & $3 u$ & $3 d$ & $3 u$ & $3 d$ & $3 u$ & $3 d$ & $3 u$ & $8,12,2, \mathrm{dd}$ \\
\hline 12 & $4 u$ & $4 d$ & $4 u$ & $4 d$ & $4 u$ & $3 u$ & $4 u$ & $3 u$ & $3 d$ & $3 u$ & $3 d$ & $3 u$ & $3 d$ & $3 u$ & $3 d$ & $3 u$ & $3 d$ & $3 u$ & $3 d$ & $3 u$ & $12,8,1, \mathrm{uu}$ \\
\hline 13 & $4 u$ & $4 d$ & $4 u$ & $4 d$ & $4 u$ & $4 d$ & $3 d$ & $3 u$ & $3 d$ & $3 u$ & $3 d$ & $3 u$ & $3 d$ & $3 u$ & $3 d$ & $3 u$ & $3 d$ & $3 u$ & $3 d$ & $3 u$ & $10,10,0,--$ \\
\hline
\end{tabular}


In polycrystalline manganites, the ordered chains separated by disordered chains may be expected to be distributed uniformly in space and the properties of 3-D manganites may be described by the bundle of chains in the direction it is investigated. In polycrystalline materials, the properties may be isotropic, but in crystals, there may be directionalities. There may be need to consider additional lateral interaction in 3-D manganites. One thing has to be pointed out here that according to the model of Luttinger [26], in one-dimensional system, spin and charge are separated. But in the present case, one dimensional manganite is simply a thread of three dimensional manganite chosen here for discussion to drive home the points more clearly.

Summary. Three important features of manganites have been investigated here: 1) correlation between electrical resistivity and magnetization in the same temperature range at different hole concentrations (x-values); 2) arrangement of $\mathrm{Mn}^{3+}$ and $\mathrm{Mn}^{4+}$ ions for different $\mathrm{x}$-values; and 3) the way the electric current flows through manganites by means of double exchange mechanism. The first feature has been investigated in three dimensional manganites and the last two in one-dimensional case for the sake of clear understanding of problems and then they have been generalized. The first problem suggests that before Tc, magnetization and resistivity are inversely proportional and beyond Tc, they are directly proportional. The second problem gives us idea about the electrical and magnetic characteristics of the material and the third problem suggests how $\mathrm{u}$ (parallel to the magnetic field) and d (antiparallel to the magnetic field) spins connected by superexchange are affected when double exchange of electrons from $\mathrm{Mn}^{3+}$ and $\mathrm{Mn}^{4+}$ ions takes place any where in the linear chain. This study may be extended for understanding the behaviours of other manganites of large and small bandwidths if magnetization and resistivity as a function of temperature at various magnetic fields and phase diagram of other manganites are available.

\section{REFERENCES}

[1] A. J. Millis, P. B. Littlewood and B. I. Shraiman, "Double Exchange Alone Does Not Explain the Resistivity of LaSrMnO," Physical Review Letters, Vol. 74, No. 25, 1995, pp. 5144-5147. doi:10.1103/PhysRevLett.74.5144

[2] A. J. Millis, B. I. Shraiman and R. Mueller, "Dynamic Jahn-Teller Effect and Colossal Magnetoresistance in $\mathrm{La}_{1-\mathrm{x}} \mathrm{Sr}_{\mathrm{x}} \mathrm{MnO}_{3}$," Physical Review Letters, Vol. 77, No. 1, 1996, pp. 175-178. doi:10.1103/PhysRevLett.77.175

[3] S. K. Sarker, "Phase Transition in the Double Exchange Model: A Schwinger Boson Approach," Journal of Physics: Condensed Matter, Vol. 8, No. 37, 1996, pp. L515L521.

[4] E. Dogotto, S. Yunoki, A. L. Maluezzi, A. Morento and H.
$\mathrm{Ju}$, "Ferromagnetic Kondo Model for Manganites: Phase Diagram, Charge Segregation and Influence of Quantum Localized Spins," Physical Review, Vol. 58, No. 10, 1998, pp. 6414-6427.

[5] E. Dogotto, T. Hotta and A. Moreo, "Colossal Magnetoresistant Materials: The Key Role of Phase Separation," Physics Reports, Vol. 344, No. 1-3, 2001, pp. 1-153. doi:10.1016/S0370-1573(00)00121-6

[6] Y. Tokuro, "Critical Features of Magnetoresistive Manganites," Reports on Progress in Physics, Vol. 69, No. 3, 2006, pp. 797-851. doi:10.1088/0034-4885/69/3/R06

[7] L. P. Gorkov and V. Z. Kresnin, "Mixed Valence Manganites, Fundamental and Main Properties," Physics Reports, Vol. 400, No. 3, 2004, pp. 149-208. doi:10.1016/j.physrep.2004.08.003

[8] M. B. Salamon and M. Jaime, "The Physics of Manganites: Structure and Transport," Reviews of Modern Physics, Vol. 73, No. 3, 2001, pp. 583-628. doi:10.1103/RevModPhys.73.583

[9] E. Dogotto, "Open Questions in CMR Manganites, Relevance of Clustered States and Anologies with Other Compounds including the Cuprates," New Journal of Physics, Vol. 7, 2005, pp. 67-95. doi:10.1088/1367-2630/7/1/067

[10] C. N. R. Rao and R. Reveau, "Crystal Structure of Ferromagnetic Insulated $\mathrm{La}_{1-\mathrm{x}} \mathrm{Ca}_{\mathrm{x}} \mathrm{MnO}_{3}$," World Scientific, Singapore City, 1998.

[11] T. V. Ramkrishnanm, H. R. Krishnamurthy, S. R. Hasan and G. V. Pai, "The Theory of Insulator Metal Transition and Colossal Magnetoresistance in Doped Manganites," Physical Review Letters, Vol. 92, No. 15, 2004, Article ID: 157203. doi:10.1103/PhysRevLett.92.157203

[12] H. R. Krishnamurthy, "A New Theory of Doped Manganites Exhibiting Colossal Magnetoresistance," Pramana: Journal of Physics, Vol. 64, No. 6, 2005, pp. 1063-1074. doi:10.1007/BF02704168

[13] W. E. Picket and D. J. Singh, "Comment on: Density Functional Distribution of the Electronic Structure of $\mathrm{LaMnO}_{3}$ $(\mathrm{M}=\mathrm{Sc}, \mathrm{Ti}, \mathrm{V}, \mathrm{Cr}, \mathrm{Mn}, \mathrm{Fe}, \mathrm{Co}, \mathrm{Ni})$, , Physical Review, Vol. B53, 1996, pp. 1146-1160.

[14] G. Li, H.-D. Zhou, S. J. Feng, X.-J. Fan, X.-G. Li and Z. D. Wang, "Competition between Ferromagnetic Metallicand Paramagnetic Insulating Phases in Manganites," Journal of Applied Physics, Vol. 92, No. 3, 2002, pp. 1406-1410. doi:10.1063/1.1490153

[15] S. Khan and R. J. Singh, "Temperature Profile of Resistivity of Manganites," Modern Physics Letters B, Vol. 21, No. 26, 2007, pp. 1795-1805. doi:10.1142/S0217984907014127

[16] P. Schiffer, A. P. Ramirez, W. Bao and S.-W. Cheong, "Low Temperature Magnetoresistance and Magnetic Phase," Physical Review Letters, Vol. 75, No. 18, 1995, pp. 33363339. doi:10.1103/PhysRevLett.75.3336

[17] D. Q. Liao, Y. Sun, R. F. Yang, Q. A. Li and Z. H. Cheng, "Spontaneous Magnetization and Resistivity Steps in the Bilayered Manganite $\left(\mathrm{La}_{0.5} \mathrm{Nd}_{0.5}\right)_{1.2} \mathrm{Sr}_{1.8} \mathrm{Mn}_{2} \mathrm{O}_{7}$," Physical Review B, Vol. 74, 2006, Article ID: 174434.

[18] C. Zener, "Interaction between the D-Shells in the Transition Metals. II. Ferromagnetic Compounds of Manganese 
with Perovskite Structure," Physical Review, Vol. 82, No. 3, 1951, pp. 403-405. doi:10.1103/PhysRev.82.403

[19] M. H. Tsai, Y. H. Tang, H. Chou and W. I. Wu. <arxiv,org|pdf|Cond-mat|0604367>

[20] P. W. Anderson, "Antiferromagnetism. Theory of SuperExchange Interaction," Physical Review, Vol. 79, No. 2, 1950, pp. 350-356. doi:10.1103/PhysRev.79.350

[21] J. B. Goodenough, "Theory of the Role of Covalence in the Perovskite Type Manganites [La, $M(\mathrm{II})] \mathrm{MnO}_{3}$," Physical Review, Vol. 100, No. 2, 1955, pp. 564-573. doi:10.1103/PhysRev.100.564

[22] J. B. Goodenough, "An Interpretation of the Magnetic Properties of the Perovskite Type Mixed Crystals $\mathrm{La}_{1-\mathrm{x}} \mathrm{Sr}_{\mathrm{x}} \mathrm{CoO}_{3-\lambda}$," Journal of Physics and Chemistry of Solids, Vol. 6, No. 2-3, 1958, pp. 287-297. doi:10.1016/0022-3697(58)90107-0
[23] J. Kanamori, "Superexchange Interaction and Symmetry Properties of Electron Orbitals," Journal of Physics and Chemistry of Solids, Vol. 10, No. 2-3, 1959, pp. 87-98. doi:10.1016/0022-3697(59)90061-7

[24] P. Raychaudhuri, K. Sheshadri, P. Taneja and S. Bandophadhyay, "Spin Polarized Tunnelling in the Half Metallic Ferromagnets $\mathrm{La}_{0.7-\mathrm{x}} \mathrm{Ho}_{\mathrm{x}} \mathrm{Sr}_{0.3} \mathrm{MnO}_{3}$ ( $\mathrm{x}=0$ and 0.15): Experiment and Theory," Physical Review B, Vol. 59, 1999, pp. 13919-13926.

[25] A. de Andres, M.-G. Hernandez and J. I. Martinetz, "Conduction Channels and Magnetoresistance in Polycrystalline Manganites," Physical Review B, Vol. 60, 1999, pp. 7328-7334.

[26] J. M. Luttinger, “An Exactly Soluble Model of a Many Fermion System," Journal of Mathematical Physics, Vol. 4, No. 9, 1963, pp. 1154-1162. doi:10.1063/1.1704046 